\title{
Digital Transformation: Harnessing Digital Technologies for the Next Generation of Services
}

\begin{tabular}{|r|l|}
\hline Journal: & Journal of Services Marketing \\
\hline Manuscript ID & JSM-01-2019-0034 \\
\hline Manuscript Type: & Commentary \\
\hline Keywords: & $\begin{array}{l}\text { Big Data, digital economy, Digital, Customer Service, customer value, } \\
\text { service encounters }\end{array}$ \\
\hline \multicolumn{2}{l}{} \\
\end{tabular}

\section{SCHOLARONE \\ Manuscripts}




\title{
Digital Transformation: \\ Harnessing Digital Technologies for the Next Generation of Services
}

\begin{abstract}
:
Purpose: The purpose of this commentary is to discuss digital transformation and its four trajectories- digital technology, digital strategy, customer experience and datadriven business models that could shape the next generation of services. Understanding where opportunities for digital service innovation lie, how best to create and capture value, whether both the market and organisations are all ready for this digital change.
\end{abstract}

Design/methodology/approach: Providing services is a proven and effective way to secure a competitive position, deliver long-term stable revenues and open up new market opportunities. However, it is also clear that some organizations are struggling to digitally transform. Therefore, the commentary provides a brief insight into how firms explore the possibilities of digital transformation and navigate these uncharted waters.

Findings: Today's digital technologies affect the organization outside and in, enabling the creation of new business models and transforming the customer experience. The incumbents are acutely aware that they need to transform strategically - to build new networks and value chains.

Originality/value: This commentary extends earlier work exploring the digital disruption within services to highlight a number of connected areas: the challenges and opportunities of digital transformation at a strategic level, as well as, understanding and enhancing the customer experience and seeing how new datadriven business models can underpin service transformation.

Keywords: Digital transformation strategy, service, customer experience, business model, data-driven business models, strategy, machine learning, AI, digital technologies 


\section{Introduction:}

Customer expectations in both the business to business (B2B) and business to consumer (B2C) worlds are being ramped up as market entrants endlessly innovate to enhance their services. But companies can also see the opportunities that are being created in this new experience and platform-led economy. At the same time, the fear of new competition emerging from unexpected quarters is very real. Ultimately, firms are terrified of the Kodak and Toys R Us scenarios: eyes fixed on a game-changing technology as it comes over the horizon but unable to make the organisational changes that would allow them to embrace it. But the Kodak example is instructive in lots of ways. It is very difficult for large companies to achieve the kind of shift from product to service transformation demanded of them in the new digital era. Equally, they can't opt out.

That change has happened because firms have looked at the data they have collected over the years and by carefully analysing what it tells them, can see how to fine tune their business plans to serve their customers better and to respond to what they actually need and what they say. Collaborations between firms has also helped to create more accurate planning and while competitors will still want to out sell their rivals, they are aware that there is an interdependency within their respective ecosystems which in turn means they need to be open to new ways of working and to learning the lessons others may have taken on board through trial and error.

"Test, test, test and don't be afraid to fail" isn't just phraseology taken from a business text book, firms know that they have to have eyes in the back of their heads to see what the next wave of innovations may be in their business streams and to be adopters of these new advances somewhere down the line. If they don't want to be first, then knowing when to enter the new markets that are developing and replacing the old products and ways of working will be a critical decision for them. Workforces too will need to be responsive to change, and perhaps employees too should keep an eye out for their next job, which in all likelihood will have a very different job description from the one they undertake at the moment. They might even like to write it themselves with a foot firmly planted in the digital technology of the future. 
Newer companies are more agile to technological change as they don't inherit old systems and working practices that will at some time need to undergo change. Compare, for example, Alibaba a Chinese company that is just 18 years old, to Emirates which is 32 years old and IBM which is just over 70 years old. So how can they seize the opportunities digitalisation presents without falling prey to its disruptive tendencies? In this commentary, I will discuss four trajectories of the digital service transformation (digital technology, digital strategy, customer experience and datadriven business Models) that could shape the next generation of services.

\section{Digital Technology Trajectory}

Arguably, the nature of digital technologies is a fundamental key difference causing the dramatic shift within organizations and in the competitive landscape. Today, we are reaching an inflection point, where the effect of these digital technologies' manifests with "full force" and enables "unprecedented things" (Brynjolfsson \& Mcafee 2014). A convergence of multiple technologies, such as mobile, location-based, virtual reality, digital twins, blockchains, Artificial Intelligence (AI), wearable technologies, chatbots, neuroscience and business process automation, as well as machine-tomachine interactions through the IoT are happening today. The innovations build on and amplify one another in a fusion of multiple technologies across the physical, digital and social realms (Bolton et al. 2018). In addition, digital technologies have mobility and ubiquitous connectivity features, which provide immediate interaction and access to a wide range of data and computing power; these features are also reflected in today's products and services and enable a wider reach (of people), regardless of geographical location (Schwab 2016; Lanzolla \& Anderson 2008; Lanzolla \& Giudici 2017).

The exponential availability of data as a result of the discussed features enables companies to analyze the insights and derive the right decisions in real time. Therefore, AI is a hugely powerful tool which is already having an impact on us all. A new report from the McKinsey Global Institute has predicted that it could add $\$ 13$ trillion to the global economic output by 2030 and that around $70 \%$ of companies will have implemented some form of AI by the end of the next decade. AI - and more specifically machine learning, and even more specifically deep learning - is becoming part of our everyday lives, employed by the likes of Google to identify millions of images. Selfdriving cars are using it to learn how to recognise obstacles and react accordingly. And these deep learning systems are achieving impressive results when you measure their overall accuracy across huge datasets. But in many real-world applications it may not be enough to work well 'on average'. These systems need to be as sure as the data allows them to be, particularly when there are serious downsides to getting it wrong. 
These terms are often used interchangeably but it is helpful to understand where the distinctions lie. Artificial Intelligence (AI) is the term that tends to be most widely used. Although it's not wrong to use it as a catch-all, it's not very precise. AI is an umbrella term for anything done by a machine that mimics the way humans think. As a concept, it's been around since the 1950s. Some would say that it goes back to the ancient Greeks. Machine learning is the sub-discipline of AI which has really taken off in recent years and caused the enormous upsurge in interest in the field. This is when an algorithm is 'trained' - generally using large amounts of data - to learn how to perform a task rather than hand-coding software routines with a specific set of instructions. Deep learning is a type of machine learning that takes the technology to the next level. Modelled on neurons of the brain, it digests vast quantities of data through deep neural networks and learns from it without human interference. And just like us (only more reliably) it learns from its mistakes. In fact, many of these ideas have been around for decades but to work they needed access to the gargantuan datasets and computational power that has only become available in recent years.

Amazon Alexa provides a good example of AI technological development which is disrupting business-to-consumer markets. One of the technological challenges of developing a virtual assistant with human-computer interaction based on voicerecognition. Human vocal communication contains sophisticated and implied information that we learn to understand intuitively, but which must be unpicked for a computer to interpret. For example, if we ask Alexa "how tall is the Gherkin?", Alexa needs to interpret not only the words used, but also the meaning to know that we are referring to the height of a building rather than the length of a pickled vegetable! Alexa is the service, rather than a device. It is a virtual assistant that can be used across a range of devices and applications. Recently, Amazon patented a new Alexa feature that knows when you're ill and offers you medicine. It analyses speech and identifies other signs of illness or emotion (Cook 2018). Amazon has stimulated technology development for Alexa, by encouraging developers (in other companies or as individuals) to build and publish 'skills' using the Alexa Skills Kit. Once published, third party skills can be made available across Alexa-enabled devices. To date there are over 50,000 skills written by 325,000 developers. This is an example of how Amazon encourage innovation across ecosystems rather than relying on closed internal R\&D for innovation.

As digital technology overtakes old ways of working more firms are creating digital systems that are highly responsive and that can move goods from the factory to the consumer as soon as orders are placed. Gone are the days when factories produced goods and sales forces waited to sell them. In a B2B setting, for example, CEMEX developed an e-commerce platform called 'CEMEX Go' for a seamless service delivery of a Readymix concrete product. This is the first end-to-end digital platform in the cement industry. The implementation of the platform required more than technical development; it required digital capabilities, and a digital culture. CEMEX is working 
towards a culture in which decision makers and employees have a 'digital mindset', with the willingness and capability to develop solutions in practice that solve customer needs (CEMEX 2017). These technologies do not merely automate basic processes, satisfy information needs or affect the business strategy, as was the role of IT in previous eras (Ward et al. 1990); they also have the capacity to reformulate the entire service strategy as a result of their unprecedented capabilities: ensuring efficiency, scalability, reliability and predictability of core operations, while simultaneously facilitating rapid development and the implementation of rapid innovations in response to, or even anticipation of, customers' needs.

\section{Digital Strategy Trajectory}

It became clear that digital service transformation would be a strategic focus for firms in different industry sectors. Even if a business or a sector has yet to experience the full force of digitalisation's disruptive consequences, it is playing out in other industries. And it can mean bad news for the incumbents as new market entrants come in and move the goal posts. But how do the big multinationals approach digitalisation when the scale and complexity of organisation-wide transformation is so challenging?

After all, management consultants and practitioners have been thinking about service strategy and transformation for decades, largely in response to advances made in information technologies in the 1990s and 2000s. A 1999 paper in the Sloan Management Review, for example, talked about the need to involve the entire organisation in business transformation. Its authors argued that senior managers must change the world view of the organisation, address deeply held values and beliefs while building new skill sets and putting in place new management processes to support the new reality. However, digital service transformation is even tougher. This is because there is a fundamental difference between the scope and complexity of the previous attempts at transformation (which has been characterised as IT-enabled transformation) and today's digital transformation. Last time it was about evolution doing more or less the same things, serving the same customers with the same products and services, but doing it better. Its main aims were to improve internal integration, support the redesign of business processes and business networks and, to an extent, enable the redefinition of business scope. Now it is about all of those things and more.

If IT-enabled transformation was evolution, digital transformation is a revolution. The so-called Fourth Industrial Revolution, in fact. Why is it so different this time round? First, the technologies themselves are different. In the IT-enabled evolution, they were primarily used to become more efficient, to optimise the business by automating basic processes and speeding up the exchange of information. The combination of today's digital technologies such as social, mobile, analytics, cloud and the Internet of Things has the potential to deliver much more far-reaching change, not least from the 
blending of personal and corporate IT environments. Innovation is also happening at an unprecedented rate as these technologies converge and build on one another. Another fundamental difference is that some of these new technologies can be embedded within products. As a result, of the vast improvements in processing power, miniaturisation and the availability of wireless connectivity, new generations of smart, connected products which can provide services as well as better products - and by doing so, pave the way for the development of completely new value propositions. From a value chain perspective, smart connectivity lends itself to a new way of working with firms collaborating with their customers and other organisations (sometimes their competitors) to co-create value. It also transforms organisations' understanding of and relationships with - their customers. They can see how customers are using their products, they can predict and pre-empt problems and they can use all of this functionality to transform the customer experience.

Today's technologically-driven change affects the organisation outside and in. As companies seek to harness these new technologies they need risk-taking to become a cultural norm. For engineering-based, product-focused multinationals, in particular, this goes against everything they know which is - for good reason- all about sticking to the rules. One of the characteristics of this new reality is that both technologies and customer behaviours are changing so fast, new initiatives are never finished but remain in a state of perpetual change. Firms need to be able to handle this level of uncertainty and be able to adapt quickly to new circumstances. As with all cultural change this is easy to say and hard to do. It means a wholesale reassessment of a company's norms and values as well as major changes in organisational structures in which the conventional model of separate functional units (production, logistics, sales, finance, IT, HR etc) is no longer effective. With the blurring of roles, comes the need for crossfunctional teams. Some are recommending the creation of interdisciplinary teams bringing together IT and business expertise, headed by an operational lead. Others suggest that Chief Information Officer (CIOs) - particularly those whose roles have been to lead on IT-enablement - are not necessarily the right people to take charge of digital transformation which affects all parts of the business. Some companies are creating new board-level positions such as Chief Digital Officer to work alongside the $\mathrm{CIO}$ and Chief Marketing officer (CMO) to take care of the digital products and services at the customer interface.

Thus, if the organisational changes that took place as a result of the advances in ICT were challenging, they are as nothing compared to what organisations face today. If they are to prosper they may need new operational structures and a whole new culture. Thus, firms are acutely aware that they need to transform strategically - to build new networks and value chains. However, it's not all bad news. The rewards this time round are also that much greater. The key differences between this revolution and the last is that this time it is not just about doing more of the same, it has the potential to transform or rewrite the entire business model and the customer 
experience. While the organisational pain needed to achieve that may be acute, it promises to transform the fortunes of those that can make it happen. For example, Emirates Airline, aims to re-invent its processes using digital technology to enable a delightful travel experience. They are examining new technologies such as AI and real-time analytics and ideas to identify the opportunities they represent for both incremental improvement and disruptive change in their business model. For example, they developed a digital application which aims to reduce aircraft turnaround at Dubai International airport. Currently, it takes around 90 minutes to turnaround aircraft like B777. The standard turnaround process consists of interiors cleaning, water and toilet servicing, loading meals, refueling, maintenance checks, loading passenger luggage and cargo, and servicing the aircraft's Auxiliary Power Unit. Different data sources are accessed and integrated to track activities on a real time basis and to raise alarms when there are delays. This enables Emirate to identify root causes and reduce potential delays (Mark Caswell 2018). But the critical questions are: how do you create new value propositions for your customers that will deliver a return on investment? How do you change from a rules-based culture to one that supports innovation and takes risks? And how do you do all that while running your existing business and keeping today's customers happy? These questions are not going away any time soon, and academia and industry will firmly focus on digital service transformation theme to address these challenges.

\section{Customer Experience Trajectory}

Being able to see your firm through the eyes of your customer is fundamental to successful service delivery. We are already seeing many examples of firms competing on customer experience, whether it's Lenovo's quick response time policy, or Disney personalising its customer interactions or omnichannel retail. Recently, the Coca-Cola Company has launched a new vending machine in China where consumers can purchase beverages, as well as return and recycle used bottles and cans. The vending machine uses AI technology such as facial recognition and sound interaction to provide an interactive experience to the consumers. Consumers who buy a beverage bottle may receive a reminder along the lines of: "you owe me a bottle, don't forget to pay back," via their mobile devices, consumers can also earn credits by recycling more. These credits can be used to send e-vouchers to friends for drinks or to purchase items made from recycled plastic (Haigh 2018).

But is this something that need to concern B2B companies? The answer is emphatically - yes. A McKinsey report in March 2016 pointed out that B2B customerexperience index ratings significantly lag behind those of retail customers. Yet B2B customer expectations are rising fast. As we now run our personal lives through smart, real- time services and customer-friendly apps, we will become increasingly unimpressed by a lack of service innovation in the workplace. And for B2B, where the value of each customer tends to be high, customer loyalty is paramount. At the same 
time, however, the notion of a B2B customer is complicated. Where in B2C a customer is usually one person, in $\mathrm{B} 2 \mathrm{~B}$ purchasing decisions are usually made by multiple stakeholders. In this scenario, understanding the customer experience is even more critical and needs sophisticated analytical tools.

This sounds like common sense. Why, then, are so many firms unaware of what their customers think of them and dangerously complacent as a result? The reason is that lots of them do not monitor the customer experience in real time and rely on simplified, single-metric ways of measuring customer satisfaction using techniques such as Net Promoter Scores and customer satisfaction surveys. This is understandable. They are relatively easy to administer and give you a set of numbers you can present to the board. These techniques fail to provide a true insight into the customer experience they are often actively misleading, lulling firms into a false sense of security until they notice - too late - they have haemorrhaged customers. This is because there are many dimensions to customer experience which cannot be measured by a single data point. Advances in machine learning mean we can now delve more fully - and more usefully - into the customer experience. By analysing the more detailed and nuanced feedback contained in the free-text comments section of surveys, social media, reviews and CRM, managers can develop a much richer view of a customer and their interactions with a firm throughout the customer journey (Villarroel Ordenes 2014; Zaki and Neely 2018).

Another common misapprehension about customer analytics is that 'Big Data' by itself is the answer. Thanks to an increasingly digitalised value chain, firms may have huge volumes of data at their disposal. But sheer volume does not - in and of itself - deliver new insights into customer behaviour. We need to know what questions to ask and how to ask them. Which is where Customer Experience (CX) analytics come in. They are able to develop a much deeper understanding of customer decisions along the whole customer journey, not just at the point of transaction. To do this, creating a framework for interrogating the data based on a deep understanding is necessary. For example, machine learning can be used to monitor dimensions such as touchpoints, value creation elements (resources, activities, context, interactions, and customer role), cognitive responses and discrete emotions (McColl-Kennedy et al. 2018), alongside the more functional measures of how well a particular product or service performs. Once these dimensions have been defined, they can then be used to interrogate the data using machine learning to score customers on only those aspects of the transaction that matter to them. This enables firms to properly identify critical pain points, unmask underlying sources of friction at the touchpoints along the customer journey and provide insights into how and where firms need to implement change to improve their responsiveness. Understanding, managing and measuring customer experience is vital for service providers. These new analytical approaches will enable firms to develop more bespoke and more responsive services and, by doing so, help to 'lock in' their loyal customers. 


\section{Data-Driven Business Models (DDBM) Trajectory}

Capitalizing on the data explosion from different systems and service encounters is increasingly becoming a necessity for businesses who wish to remain competitive. As a consequence, many firms are looking to develop new business models - effectively data-driven business models (DDBMs) (Hartmann et al. 2016). The challenges are threefold: i) how to extract data, ii) how to refine it, and iii) how to ensure it is utilized most effectively. Businesses and other organizations that fail to align themselves with data-driven practices risk losing a critical competitive advantage and, ultimately, market share and the accompanying revenue. For today's businesses, effective data utilization is concerned not only with competitiveness, but arguably survival itself. However, such innovation is notoriously difficult - particularly for large existing firms who have to contend with ingrained company structure, culture and traditional revenue streams. It is the competitive advantage associated with effective big data utilization that is driving existing mainstream businesses to become data-driven.

In some industries, such as publishing, big data has spawned entirely new business models. The shift towards a digitally oriented distribution model produced dwindling advertising revenues, but as a response, certain publisher began to accumulate data relating to their online users - users whose demographic was particularly attractive to advertisers. This data was then sold, enabling targeted and more effective advertising and thus increasing revenue. A second general industry example is in the financial services sector where trading algorithms are applied to analyze huge quantities and varieties of data enabling the capture of value in milliseconds. It is unsurprising that 71 per cent of banking firms directly report that the use of big data provides them with a competitive advantage (Turner at al. 2013) - each often finding a slightly different angle for data application. Welovroi- A start-up web application that uses big data in a way that allows marketers to directly measure the effectiveness of digital marketing campaigns and offers its services to customers in exchange for a subscription fee. Business start-ups may be inclined to utilize a usage fee or a subscription in their revenue model as it is a consistent payment and an effective way for a start-up to maintain liquid capital. Next Big Sound- A start-up organization who utilize large quantities of free available data and acquired data in the form of sales, social media interactions and purchasing data to improve the effectiveness in which a music artist is developed, produced, branded and marketed to the public. These short case examples illustrate the range of big data applications, but raise an interesting issue, namely what are the underlying questions that enable firms to capitalize on data and create data-driven business models. There are six key questions that organisations creating data-driven business models need to grapple with. First, is the question of the target outcome - what are we trying to achieve with big data? and how can this create value for customers? Take Zara, for example. They are using big data to monitor fashion trends so they can create a highly responsive supply chain that is in synch with 
the latest fashions and trends. Second, one has to ask - what is our desired offering? In essence Zara uses big data to enable their desired offering - getting the right clothing to their customers at the time they want it. Having considered both the outcome and the desired offering, the third question to ask is what data sources do we need to access? For Zara, data sources include internal data- supply chain and inventory records, as well as externally created data harvested from social media. The next stage involves asking how will we utilize these data? and how will we monetize them? Considering what analysis is required and how this analysis delivers insight that will create value. Finally, firms have to ask what are the inhibitors - the barriers that will prevent us from achieving our goal?

In order for a business to effectively utilize big data it is vital that its aims are clear and realistically attainable. Often an organization understands the potential value and benefit associated with data but fails to determine a specific aim before undertaking a time-consuming and costly data acquisition and analysis process. By identifying a predetermined target, the business can retain its focus on a desired and realistic goal and reduce unnecessary monetary and human resource wastage during the process. Furthermore, a business must decide in what way the business model will benefit the company's current offering or, alternatively, create an entirely new one. Established businesses have a tendency to utilize data to improve or enhance their current customer offering, which is often called a 'value proposition' (Chesbrough and Rosenbloom 2002; Osterwalder 2004; Johnson and Christensen 2008). It therefore follows that the value proposition is the value created for customers through the offering. A company can offer raw data that is primarily 'a set of facts' without an attached meaning. When data has been interpreted it becomes information or knowledge. Typically the output of any analytics activity attaches some insight or application.

Organizations are not restricted to a single offering. Established organizations, in particular, tend to have multiple customer offerings. But, organizations have to identify with those whom these offerings should target. There are several ways to segment customers. However, the most generic classification was used, dividing target customers into businesses (B2B), individual consumers (B2C) (Morris et al. 2005) and consumer to consumer (C2C), which is defined as facilitating the use of customers to acquire further customers. In many cases, companies could target businesses and individual consumers. On the other hand, business start-ups that do not have the luxury of traditional revenue streams tend to create an entirely new offering. For example, the start-up Farmlogs offers a service to farmers that streamlines crop and fertilizer input with satellite monitoring and weather and produces pricing patterns, increasing efficiencies throughout the farming process, thus enabling farmers to reduce unnecessary costs and improve practices and ultimately increasing revenues. Data is obviously fundamental to new generations of business models. Deciding which data is likely to be most beneficial, and the nature of that data's acquisition, is pivotally 
important to the success of business models. Established businesses with a substantial number of customers, and therefore potential customer interaction points, are well positioned to effectively utilize customer-provided data within their business models, although this data is often combined with data from other sources. This high utilization of all available data sources by established organizations is indicative that these organizations understand the value of data and orient themselves towards becoming data-driven. In particular, telecommunications and retail placing particular emphasis on self-generated data - probably due to their industry-specific customer interactions. Customer-provided data is utilized and regarded as important across all of the analyzed sectors, which is suggestive of established business organizations viewing data as a source of leverage. For example, the fashion retailer Topshop combines customer-provided data, free available data from fashion blogs and social media and existing data within its own databases when running predictive and descriptive analytics protocols to determine emerging trends within the highly competitive retail clothing industry. Without these processes in place to manage and capitalize upon the valuable source of potential customer insight, the available data, fashion retailers would lose out on significant revenue opportunities. Although start-up companies have the advantage of a 'clean sheet' when constructing data-driven business models, but also the disadvantage that they rarely have the luxury of a high number of recordable customer interaction points that can be utilized to generate revenue streams.

Methods of processing reveal the true value contained within data. Knowing which key activities will be utilized to process data enables the business to plan accordingly, ensuring that the necessary hardware, software and employee skill sets are in place. To develop a complete picture of the key activities, the different activities were structured along the steps of the 'virtual value chain' (Rayport and Sviiokla 2005). To gather data, a company can either generate the data itself internally or obtain the data from any external source (data acquisition). The generation can be done in various ways, either manually by internal staff, automatically through the use of sensors and tracking tools (e.g. Web-tracking scripts) or using crowd-sourcing tools. Insight is generated through analytics, which can be subdivided into: descriptive analytics, analytics activities that explain the past; predictive analytics, which predict/forecast future outcome; and prescriptive analytics, which predict future outcome and suggest decisions (Sivarajah et al. 2017)

Without the target of a quantifiable benefit to a business it is difficult to justify the business model construction and implementation. Incorporating a revenue model into a data-driven business model is integral to its operational success. Seven revenue streams are identified by Hartmann et al. (2016): asset sale, giving away the ownership rights of goods or service in exchange for money; lending/renting/leasing, temporarily granting someone the exclusive right to use an asset for a defined period of time; licensing, granting permission to use a protected intellectual property like a patent or 
copyright in exchange for a licensing fee; a usage fee is charged for the use of a particular service; a subscription fee is charged for the use of the service; a brokerage fee is charged for an intermediate service; or advertising. Revenue models associated with a DDBM differ considerably from a standard subscription fee such as The New York Times for advertising. These models vary considerably between sectors and within industries.

The variation among revenue models is much more substantial within established businesses, The Times is a good example of this. The current CEO realized that as physical readership continued to decline, thus reducing revenues, a unique aspect of the company was its access to a particularly high calibre of readership. With its online offering continuing to expand, it was decided that the company would offer its content online for free - although its competitors charged their online readers. With no-cost access, online readers of The Times browsed the website freely and each click and article read logged and tied the individual user preferences through his or her account. Descriptive analytics allowed The Times to build a profile unique to each reader, enabling them to be targeted by advertisers both on and off The Times website and charged at a premium because of the attractive demographic of the readers.

The revenue model for business start-ups is dominated almost entirely by either usage fee or subscription fee. The Times example shows how a business must become adaptive to the ever-changing environment within which it sits. As current technologies improve and new technologies emerge, the effect on markets, industries and individual businesses are often unforeseen and difficult to predict. Through the use of industry-focused innovation platforms, businesses can assess their individual position and look to capitalize upon new and emerging business opportunities.

\section{Conclusion}

Digital transformation has gained global attention in various service industries, due to the pervasive nature and proliferation of recent digital technologies. The world is undergoing a technological revolution. Technologies such as Artificial intelligence (AI) will transform the way we live and work, from the way we diagnose and treat cancer to the security of online transactions. This fourth industrial revolution is of a scale, speed and complexity that

is unprecedented. These technologies become critical for companies to respond to market dynamics accurately and quickly. It is changing consumer behaviour, changing the way companies are organized and changing the role of "humans" in the marketplace. The degree of complexity in digital transformations exceeds that of other previous transformations, such as IT-enabled transformation. Thus, companies are experimenting with digital technologies and they build a digital service strategy to enhance their competitive positioning which does not solely depend on the technologies they adopt, but more importantly builds on the strategy they deploy. Companies have to design the right experience for their customers. But also, deal with 
digital transformation challenges and obstacles (e.g. leadership, culture, institutional, etc.) that might stand in the way of a successful implementation. Finally, companies are looking to rewrite their business models to be more a data-driven business model (DDBMs).

\section{References:}

Bolton, R. N., McColl-Kennedy, J. R., Cheung, L., Gallan, A., Orsingher, C., Witell, L., and Zaki, M. 2018. "Customer Experience Challenges: Bringing Together Digital, Physical and Social Realms." Journal of Service Management, 29(5), pp.776-808.

Brynjolfsson, E. \& Mcfee, A., 2014. The Second Machine Age. New York, N.Y.: W.W. Norton \& Company, Inc.

Caswell, M. 2018. "New Emirates app aims to reduce aircraft turnaround delays", available at https://www.businesstraveller.com/business-travel/2018/12/27/newemirates-app-aims-to-reduce-aircraft-turnaround-delays/ (accessed 26 January 2019).

CEMEX. 2017.“CEMEX delivers the future: CEMEX Go”, available at: https://www.cemex.com/media/press-releases/Lasset publisher/nnqyAoJ3dM7x/content/cemex-delivers-the-future-cemex-go (accessed 26 January 2019).

Chesbrough, H., Rosenbloom, R. (2002) 'The Role of the Business Model in Capturing Value from Innovation.' Industrial and Corporate Change, pp.529-555.

Cook, J., 2018. The Telegraph, "Amazon patents new Alexa feature that knows when you're ill and offers you medicine", available at:

https://www.telegraph.co.uk/technology/2018/10/09/amazon-patents-new-alexafeature-knows-offers-medicine/

Haigh, L. 2018, Foodingredientsfirst, "Welcome to the future? Coca-Cola China integrates retail, recycling and AI technology in vending machine", available at: https://www.foodingredientsfirst.com/news/welcome-to-the-future-coca-cola-chinaintegrates-retail-recycling-and-ai-technology-in-vending-machine.html (accessed 26 January 2019).

Hartmann, P. M., Zaki, M., Feldmann, N., and Neely, A. 2016. "Capturing Value from Big Data - a Taxonomy of Data-Driven Business Models Used by Start-up Firms," International Journal of Operations \& Production Management (2020). 
Johnson, M. Christensen, M. "Reinventing Your Business Model." Harvard Business Review (2008) 1-9.

Lanzolla, G. \& Anderson, J., 2008. "Digital Transformation". Business Strategy Review, Summer, pp.73-76.

Lanzolla, G. \& Giudici, A., 2017. "Pioneering strategies in the digital world. Insights from the Axel Springer case". Business History, 59(5), pp.744-777.

McColl-Kennedy, J. R., Zaki, M., Lemon, K. N., Urmetzer, F., \& Neely, A. (2018), "Gaining Customer Experience Insights That Matter," Journal of Service Research, forthcoming.

McKinsey Global Institute, 2018. "Notes from the AI frontier: Modeling the impact of AI on the world economy", available at: https://www.mckinsey.com/featuredinsights/artificial-intelligence/notes-from-the-ai-frontier-modeling-the-impact-of-aion-the-world-economy (accessed 26 january 2019).

McKinsey Report. 2016. "Improving the business-to-business customer experience", available at: https://www.mckinsey.com/business-functions/marketing-andsales/our-insights/improving-the-business-to-business-customer-experience (accessed 24 January 2019).

Morris, M., Schindenhutte, M., Allen, J. "The Entrepreneur's Business Model: Toward a Unified Perspective." Journal of Business Research (2005) 725-735.

Osterwalder, A. "The Business Model Ontology - A Proposition in a Design Science Approach." PhD paper (2004).

Rayport, J., Sviokla, J. "Exploiting the Virtual Value Chain". Harvard Business Review (1995) 75-85.

Schuchmann, D. \& Seufert, S., 2015. Corporate Learning in Times of Digital Transformation: A Conceptual Framework and Service Portfolio for the Learning Function in Banking Organisations. $J A C, 8(1)$, pp.31-40.

Sivarajah, U., Kamal, M. M., Irani, Z., and Weerakkody, V. 2017. "Critical Analysis of Big Data Challenges and Analytical Methods," Journal of Business Research (70), pp. 263286. 
Turner, D.; Schroeck, M., Shockley, R. 'Analytics: The Real-World use of Big Data in Financial Services.' IBM Global Business Services (2013) 1-12.

Ward, J.M., 1987. Integrating Information Systems into Business Strategies. Long Range Planning, Vol. 20(3), pp.19-29.

Villarroel Ordenes, Francisco, Babis Theodoulidis, Jamie Burton, Thorsten Gruber, and Mohamed Zaki. (2014), "Analyzing Customer Experience Feedback Using Text Mining: A Linguistics-Based Approach." Journal of Service Research vol. 17 (3), pp. 278-295.

Zaki M. and Neely A. (2018), "Customer Experience Analytics: Dynamic CustomerCentric

Model," in Maglio P., Kieliszewski C., Spohrer J., Lyons K., Patrício L., Sawatani Y. (eds) Handbook of Service Science, Volume II. Service Science: Research and Innovations in the Service Economy, Springer, Cham. 